\title{
Scoring Systems of Peritoneal Dissemination for the Prediction of Operative Completeness in Advanced Ovarian Cancer
}

\author{
SATOSHI HOSOYA ${ }^{1}$, KAZU UEDA ${ }^{1,2}$, SUGURU ODAJIMA $^{1,3}$, KOHEI OGAWA $^{4}$, HIROMI KOMAZAKI ${ }^{1}$, \\ TOSHIYUKI SEKI ${ }^{1}$, MASATAKA TAKENAKA ${ }^{1}$, MOTOAKI SAITO ${ }^{1}$, HIROSHI TANABE ${ }^{1,3}$, KYOSUKE YAMADA $^{1}$, \\ HIROKUNI TAKANO ${ }^{1}$, YASUSHI IIDA ${ }^{1}$, NOZOMU YANAIHARA ${ }^{1}$ and AIKOU OKAMOTO ${ }^{1}$ \\ ${ }^{1}$ Department of Obstetrics and Gynecology, The Jikei University School of Medicine, Tokyo, Japan; \\ ${ }^{2}$ Department of Gynecologic Oncology, International University of Health and Welfare Mita Hospital, Tokyo, Japan; \\ ${ }^{3}$ Department of Gynecology, National Cancer Center Hospital East, Chiba, Japan; \\ ${ }^{4}$ Center for Maternal-Fetal, Neonatal and Reproductive Medicine, \\ National Center for Child Health and Development, Tokyo, Japan
}

\begin{abstract}
Background/Aim: We investigated the predictive value of scoring systems of peritoneal disseminations for complete surgery $(C S)$ at primary debulking surgery $(P D S)$ in advanced ovarian cancer. Patients and Methods: We retrospectively enrolled eligible patients with clinical stages III or IVA selected for PDS from January 2015 to December 2019. Concern variables were predictive index value (PIV) and peritoneal cancer index (PCI) from operative and pathological reports. Primary endpoints were cutoffs to predict operative completeness using the receiver operating characteristic curve. Results: Among 111 patients, $P I V \geq 8$ and $P C I \geq 13$ were the best predictors of incomplete PDS, including optimal and suboptimal surgeries (AUC $=0.821$ and 0.855 , respectively). $C S$ rates in $P I V \leq 6$ and $P C I \leq 12$ were significantly higher than in $P I V \geq 8(89.3 \%$ vs. $47.2 \% ; p<0.05)$ and $P C I \geq 13(90.9 \%$ vs. 41.2\%: $p<0.05)$. Conclusion: PIV and PCI are potential predictors for $C S$ at $P D S$.
\end{abstract}

Every year, numerous patients suffer from ovarian cancer, the seventh most prevalent malignant tumor in women, with almost 240,000 newly diagnosed cases annually, as well as the eighth most common cause of female cancer death, with almost 150,000 deaths annually worldwide (1). In Japan, the incidence of ovarian cancer has been increasing up to 15.0

This article is freely accessible online.

Correspondence to: Kazu Ueda, Department of Gynecologic Oncology, International University of Health and Welfare Mita Hospital, 1-4-3 Mita, Minato-ku, Tokyo 108-8329, Japan. Tel: +81 334518121, Fax: +81 334540067, e-mail: kazu@jikei.ac.jp

Key Words: Advanced ovarian cancer, complete surgery, primary debulking surgery, predictive index value, peritoneal cancer index. per 100,000 women, almost half of whom were newly diagnosed as advanced ovarian cancer (AOC) with International Federation of Gynecology and Obstetrics (FIGO) stage III and IV (36.2\% and 10.0\%, respectively) (2).

Complete resection in primary debulking surgery (PDS) is considered the most favorable therapeutic option for AOC (3). Recently, several randomized control trials (RCTs) compared the postoperative prognosis between PDS and interval debulking surgery (IDS) after neoadjuvant chemotherapy (NAC) (4-7). Of these trials, the EORTC and CHORUS studies showed non-inferiority of IDS to PDS (4, 5). In the SCORPION trial, NAC + IDS was not superior to PDS in improving prognosis, but the authors considered $\mathrm{NAC}+$ IDS as an acceptable strategy for patients with large dissemination burdens because the incidence of operative complications was less than that for PDS (7). Although other RCTs are ongoing to clarify therapeutic superiority of PDS to overcome the limitations in previous studies, such as the low rate of complete surgery $(8,9)$, gynecologists need to pursue complete surgery in PDS (complete PDS), and if it seems difficult, consider NAC + IDS. Therefore, in deciding on operative options for AOC, a reliable predictive model for operative completeness in PDS based on preoperative examinations should be established.

Recently, diagnostic laparoscopy has been indicated as the safest method for tumor biopsy in AOC cases, and it has been also established as a potential strategy for detecting cases with large disseminations likely to result in suboptimal surgery in PDS (10). Furthermore, some quantitative scoring systems for abdominal disseminations, such as predictive index value (PIV) and Peritoneal Cancer Index (PCI), have been used to predict operative results $(11,12)$. However, the validation of their predictive value could differ between institutions, depending on factors such as the rate of complete PDS, number of surgeries of AOC, and familiarity 
with these scores. Moreover, because most of them focus on the predictive value for suboptimal surgery in PDS, any valid consensus for predicting complete PDS have not been established. Therefore, data on their value for predicting operative completeness need to be collected. Additionally, although a recent Cochrane review pointed out that the laparoscopic predictive models resulted in suboptimal surgery at PDS in some cases, even after a diagnostic laparoscopy indicated that optimal debulking surgery was feasible (13), reports on limitations of these scores in predicting operative outcomes have been limited.

In this study, we retrospectively investigated the value and limitations of PIV and PCI for the prediction of operative completeness in PDS.

\section{Patients and Methods}

Patients. In this retrospective study, we enrolled patients who were all selected for PDS at our affiliated hospitals from January 2015 to December 2019. These patients were diagnosed with AOC, including fallopian tube and peritoneal cancers, with clinical stage III or IVA by preoperative examinations, such as computed tomography (including positron emission tomography-computed tomography), and magnetic resonance imaging. If necessary, diagnostic laparoscopy and pleural cytology were also performed. Patients who had no staging surgery, who were diagnosed with double ovarian and endometrial or cervical cancers, who underwent primary diagnostic surgery, such as hysterectomy or bilateral salpingo-oophorectomy, at a previous hospital, and who had insufficient operative or pathological reports for peritoneal dissemination were excluded. Additionally, according to our previous report (14), we conventionally selected NAC + IDS as a therapeutic option for those with extensive tumor disseminations or a tumor in the right triangular ligament identified by preoperative imaging examinations or diagnostic surgeries. We planned to gather almost 100 eligible patients, referring to previous studies $(11,15,16)$. This study was approved by the institutional review board for clinical research of the Jikei Medical School of Medicine (Approval Number: 31-234[9733]). We obtained informed consent from patients by posting the opt-out at the outpatient office of our department.

Surgical procedure. Standard PDS at our institutions usually includes hysterectomy, bilateral salpingo-oophorectomy, omentectomy, retroperitoneal lymphadenectomy, and appendectomy. If necessary, we perform peritonectomy, visceral resection, diaphragmatic procedure, hepatectomy, splenectomy, pancreatectomy, or cystectomy with the cooperation of surgeons. Complete surgery is defined as complete resection of all abdominal tumors without macroscopic residues. Optimal surgery is defined as incomplete resection of all abdominal tumors, leaving macroscopic residues $\leq 1 \mathrm{~cm}$ in maximal diameter. Additionally, suboptimal surgery is defined as incomplete surgery of all abdominal tumors, leaving macroscopic residues $\geq 1 \mathrm{~cm}$ in maximal diameter, including surgeries that were initially planned PDS but ended in probe laparotomy because of large tumor dissemination.

Predictive index value and peritoneal cancer index. We applied PIV (11) and PCI (17) (Figure 1A and B) in assessing intraoperative peritoneal dissemination, referring to operative and histopathological reports. PIV was reported by Fagotti et al. (11) in 2006 as a laparoscopic scoring system for predicting suboptimal surgery for AOC. Omental cake, peritoneal carcinomatosis, diaphragmatic carcinomatosis, mesenteric retraction, bowel and stomach infiltration, and liver metastasis are evaluated intraoperatively during diagnostic laparoscopy. If each category is positive, an index value of 2 is assigned, with a possible total score of 14 . Fagotti et al. found that PIV $\geq 8$ could predict suboptimal surgery with a positive predictive value of $100 \%$. PCI was reported by Sugarbaker et al. (15) in 1995 for the quantitative assessment of peritoneal carcinomatosis in advanced colon cancer. In PCI scoring, the abdominopelvic cavity (regions 0-8) and small bowels (regions 9-12) are divided into 13 regions. The lesion size score (LSS) in each region is then calculated. LSS 0 is defined as no visible tumor in each region. LSS 1, 2, and 3 are assigned according to the maximum size of tumor dissemination in each region, namely, $0-0.5 \mathrm{~cm}, 0.5-5 \mathrm{~cm}$, and $>5 \mathrm{~cm}$, respectively. The possible total LSS was 39 points.

Endpoints. The primary endpoints were the best cutoff values of both scores for predicting operative completeness in PDS and the rates of complete PDS in each category of both scores. The secondary endpoints were clinicopathological features between the groups under and over the cutoffs of both scores and the correlation between them. Moreover, we investigated the characteristics of cases that resulted in suboptimal surgery with lower than the two cutoffs to understand limitations of these scores in predicting operative results.

Statistical analysis. First, the receiver operating characteristic (ROC) curve was used to determine the best cutoff values for PIV and PCI to identify patients likely to have incomplete PDS (optimal and suboptimal surgery in PDS) as an alternative indicator for the prediction of complete PDS. Next, the comparison of the rates of complete surgery in each category of both scores was assessed by $\chi^{2}$ test. The correlation was assessed by Spearman's rank correlation coefficient test. Continuous data were presented as means \pm standard deviation and analyzed using Student's $t$-test. Categorical data were presented as percentages and analyzed using $\chi^{2}$ test. We considered $p$-values $<0.05$ to be statistically significant. Statistical analyses were conducted using Stata SE 14 (STATA Corp., College Station, TX, USA). Additionally, Prism 8.01 software (GraphPad Inc.) was also used for preparing column figures.

\section{Results}

Demographic and operative characteristics. A total of 111 patients were eligible for the analysis (Figure 2). Patient characteristics are shown in Table I. The mean age was $56.8 \pm 10.6$ years old. The number of patients with clinical stages IIIA, IIIB, IIIC, and IVA were 17 (15.3\%), 28 $(25.2 \%), 65(58.6 \%)$, and $1(0.9 \%)$, respectively. According to the final histopathological reports, $72(64.9 \%)$ patients had high-grade serous carcinoma and $19(17.1 \%)$ were diagnosed with clear cell carcinoma. Complete surgery was achieved in 84 patients $(75.7 \%)$, optimal surgery in 7 patients $(6.3 \%)$, and suboptimal surgery in 20 patients $(23.8 \%)$, including 16 cases ended in probe laparotomy.

Primary endpoints. The best cutoffs of both scores are shown in Figure 3, and the rates of complete PDS for each 


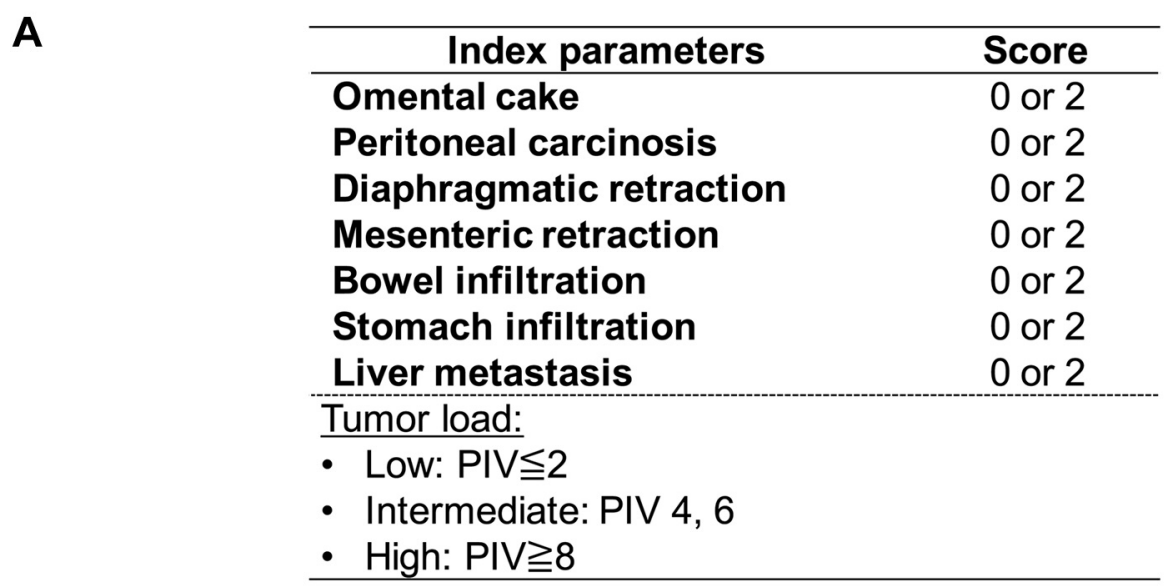
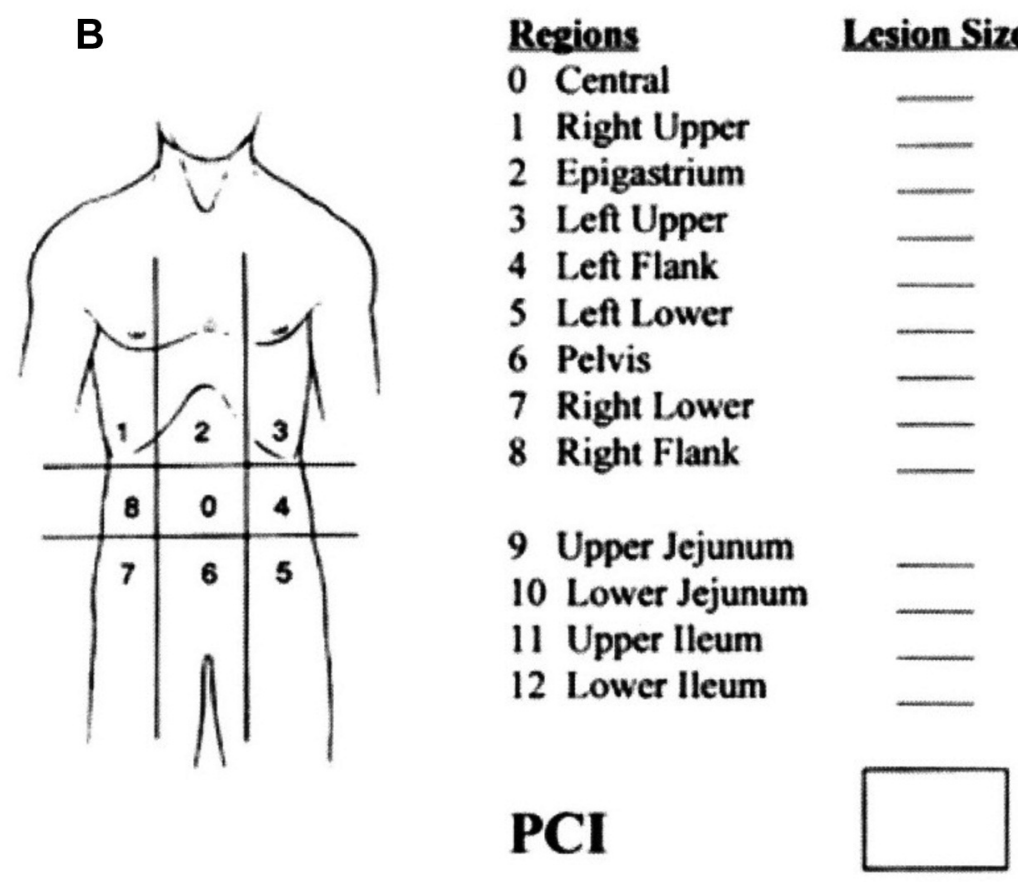

Lesion Size Score LS 0 No tumor seen

LS I Tumor up to $0.5 \mathrm{~cm}$

LS 2 Tumor up to $5.0 \mathrm{~cm}$

LS 3 Tumor $>5.0 \mathrm{~cm}$ or confluence

Figure 1. Predictive index value (PIV) (11) (A) and peritoneal cancer index (PCI) (16, 17) (B). The graphic in (B) is cited from reference \#17.

category are shown in Figure 4A and B. A PIV cutoff value $\geq 8$ was determined as the best predictor of incomplete PDS [area under the curve $(\mathrm{AUC})=0.821 ; 95 \%$ confidence interval $(\mathrm{CI})=0.731-0.911$; sensitivity, $70.4 \%$; specificity, $79.8 \%$; positive predictive value, $52.8 \%$; negative predictive value, 89.3\%] (Figure 3). The rate of complete PDS among patients with PIV $\leq 6$ was significantly higher than that in those with PIV $\geq 8$ (89.3\% vs. $47.2 \% ; p<0.05)$. Complete PDS was achieved in $64.3 \%$ of patients even with a PIV of 8 (Figure $4 \mathrm{~A})$. Additionally, a PCI cutoff value $\geq 13$ was determined as the best predictor of incomplete PDS (AUC $=0.855$; 95\% CI=0.763-0.947; sensitivity, 74.1\%; specificity, 83.3\%; positive predictive value, $58.8 \%$; negative predictive value, 90.9\%) (Figure 3). The rate of complete PDS among patients with $\mathrm{PCI} \leq 12$ was significantly higher than that in those with PCI $\geq 13$ (90.9\% vs. $41.2 \% ; p<0.05$ ). Complete PDS was 


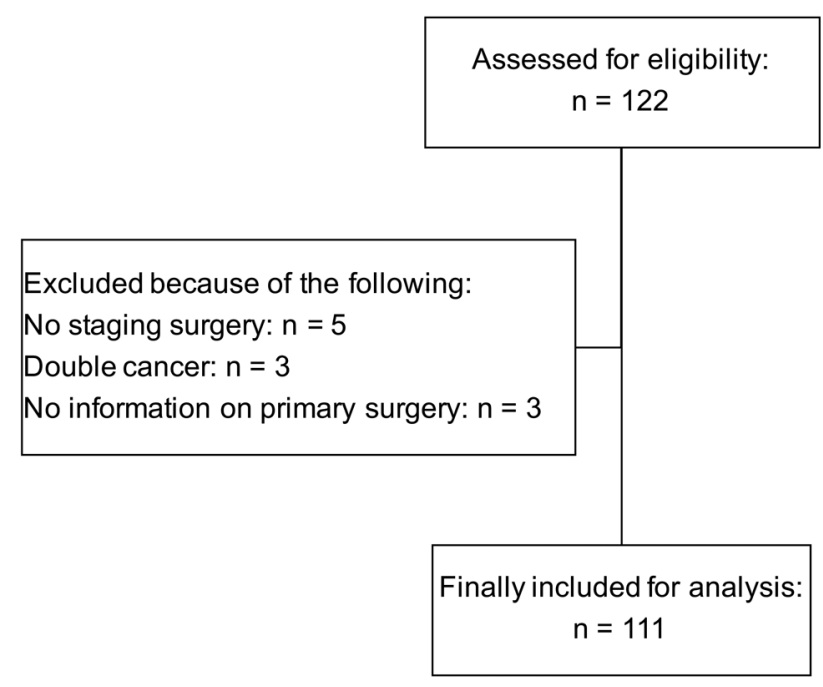

Figure 2. Flow diagram for patient selection.

achieved in $62.5 \%-70 \%$ of patients, even in those with PCI between 13 and 18 (Figure 4B).

Secondary endpoints. A strong correlation was found between PIV and PCI (correlation coefficient $=0.899 ; p<0.05$ ). The clinicopathological features of the groups under and over the cutoffs are shown in Table II and Table III. The patients with PIV $\geq 8$ or PCI $\geq 13$ had significantly higher preoperative CA125 levels, rate of positive ascites, and FIGO stage, whereas no significant differences were found in age and histology. In the further analysis, four cases ended in suboptimal surgery with lower than either cutoff for PIV or PCI. The findings of tumor disseminations in these patients are shown in Table IV. Among the four cases, three had deep tumor infiltrations to retroperitoneal organs (ureters, bladder, and vertebrae), which were not detected on preoperative imaging tests and could not be assessed by PIV and PCI. One case had widespread mesenteric retractions and disseminations in the small bowel that were not detected on preoperative imaging examinations.

\section{Discussion}

Several previous studies developed scoring systems for predicting operative results in PDS for patients with AOC $(16,18-21)$. Among them, the predictive value has been mainly focused on suboptimal surgery, and the data on their predictive values for complete PDS are controversial. Our analysis showed that PIV $\leq 6$ and PCI $\leq 12$ had reliable predictive values for complete PDS.

The study by Fagotti et al. (22) on operative outcomes in PDS and PIV concluded that, at PIV $\geq 8$, the rate of complete
Table I. Patient clinicopathological characteristics.

\begin{tabular}{lc}
\hline Characteristics & \\
\hline Total cases, $\mathrm{n}$ & 111 \\
Age (years), mean \pm SD & $56.8 \pm 10.6$ \\
Preoperative CA125 level (IU/ml), mean \pm SD & $1,894.7 \pm 3,053.2$ \\
Ascites in CT scan, n (\%) & \\
Negative & $30(27.0)$ \\
Positive & $81(73.0)$ \\
FIGO stage, n (\%) & \\
IIIA & $17(15.3)$ \\
IIIB & $28(25.2)$ \\
IIIC & $65(58.6)$ \\
IVA & $1(0.9)$ \\
Histology, n (\%) & \\
High-grade serous carcinoma & $72(64.9)$ \\
Clear cell carcinoma & $19(17.1)$ \\
Endometrioid carcinoma & $9(8.1)$ \\
Mucinous carcinoma & $3(2.7)$ \\
Other types* & $8(7.2)$ \\
Operative procedure & \\
Primary debulking surgery, n (\%) & \\
Pelvic peritonectomy & \\
Diaphragmatic peritonectomy & $95(85.6)$ \\
Whole peritonectomy & $28(25.2)$ \\
Rectectomy & $18(16.2)$ \\
Large colon resection & $51(45.9)$ \\
Small bowel resection & $22(19.8)$ \\
Retroperitoneal lymph node resection & $10(9.0)$ \\
Splenectomy & $89(80.2)$ \\
Major hepatectomy & $5(4.5)$ \\
Pancreatectomy & $1(0.9)$ \\
Ended in laparotomy & $1(0.9)$ \\
(assigned to Suboptimal surgery) & $16(14.4)$ \\
Operative outcomes, $\mathrm{n}(\%)$ & \\
Complete surgery & \\
Optimal surgery & $84(75.7)$ \\
Suboptimal surgery & $20(18.0)$ \\
\hline & \\
& \\
\hline
\end{tabular}

Continuous data are presented as means \pm standard deviation (SD); categorical data are presented as number (percentage). *Other types, including 6 carcinosarcomas and 2 low-grade serous carcinomas. CT: Computed tomography; FIGO: International Federation of Gynecology and Obstetrics staging system.

and optimal surgery was less likely at their institution. One retrospective analysis showed that PIV was not reliable in the prediction of complete PDS (AUC $=0.51$ ) (16), whereas another prospective study showed that PIV had a moderate predictive value (AUC=0.702) (23). However, our retrospective study found that PIV was highly predictive (AUC $=0.821$ ) for complete PDS and determined a cutoff value of $\leq 6$. Thus, PIV can be a valuable tool in predicting complete PDS. Regarding the operative outcomes in PDS and PCI, several studies showed that patients with PCI $\leq 10$ had a higher rate of complete PDS (over 90\%) than those with PCI $>10(16,24)$. A prospective study by Elzarkaa et al. (25) showed that the rate of complete PDS was $78.6 \%$ in 


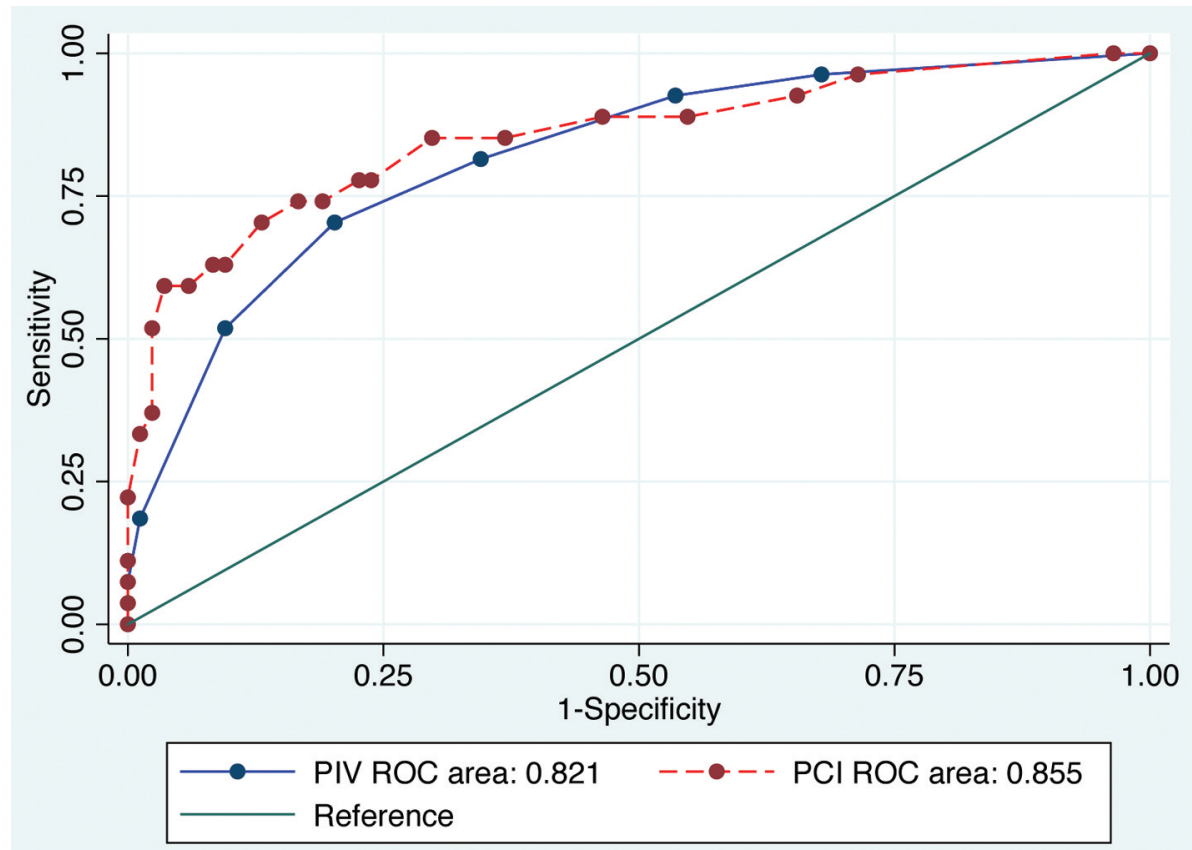

Figure 3. Optimal cutoff values of predictive index value (PIV) and peritoneal cancer index (PCI) for the prediction of incomplete PDS. A PIV cutoff value $\geq 8$ was determined as the best predictor of incomplete PDS (AUC=0.821; 95\%CI=0.731-0.911; sensitivity, 70.4\%; specificity, 79.8\%; positive predictive value, $52.8 \%$; negative predictive value, $89.3 \%$ ), whereas a PCI cutoff value $\geq 13$ was determined as the best predictor of incomplete PDS (AUC=0.855; 95\%CI=0.763-0.947; sensitivity, 74.1\%; specificity, 83.3\%; positive predictive value, 58.8\%; negative predictive value, 90.9\%). PIV: Predictive index value; PCI: peritoneal cancer index; PDS: primary debulking surgery.

patients with $\mathrm{PCI} \leq 13$, compared with $26.9 \%$ in those with PCI $>13$, similar to our results. Thus, PCI seems to be an informative tool for predicting operative results, and the data on its predictive value are consistent between our study and previous ones. According to these considerations, PIV $\leq 6$ and $10 \leq \mathrm{PCI} \leq 13$ seem to be generally useful as predictive indicators for complete PDS.

Furthermore, we found that patients with larger tumor dissemination, PIV $\geq 8$ or PCI $\geq 13$, had higher CA125 levels, rate of positive ascites, and advanced FIGO stage, which is clinically logical. Since the diagnosis and assessment of tumor dissemination in patients with stage IIIA or IIIB are not complicated, these predictive scores should be more important in those with stage IIIC. Therefore, we further conducted a sub-analysis of cases with clinical stage IIIC (Figure 5A and B). In 65 patients with stage IIIC, the rates of complete PDS with each PIV and PCI agreed with the results of the primary analysis, and over $90 \%$ of complete PDS was achieved when these scores were under the cutoffs. This sub-analysis showed that both scores were useful for predicting operative outcomes in the subgroup with advanced FIGO stage. Furthermore, although a strong correlation was found between PIV and PCI, the result of the ROC curve for PCI was a little higher than that for PIV. Similarly, two previous reports showed that PCI had a slightly higher predictive value than PIV for complete PDS (16, 23), possibly because PCI has more detailed scoring points on tumor diameter compared with PIV. However, PCI could be underestimated by approximately 2 points in diagnostic laparoscopy compared with that in laparotomy (26). Since our results were based on abdominal findings of disseminations at laparotomy, PCI in diagnostic laparoscopy should be interpreted carefully.

Diagnostic laparoscopy is performed worldwide as preoperative assessment of unresectable dissemination, such as in the small bowel. Recent reports made a consensus that diagnostic laparoscopy before PDS could decrease futile PDS resulting in suboptimal surgery $(13,27,28)$. However, the scoring systems for abdominal dissemination cannot precisely exclude some cases that ultimately resulted in suboptimal surgery, even if the systems indicated that complete and optimal surgery were feasible at PDS. Our evaluation on the limitations of PIV and PCI found four cases with PIV $\leq 6$ that resulted in suboptimal surgery, one of which had massive dissemination in the small bowel and mesentery, and the other three with deep tumor infiltration to retroperitoneal organs. To minimize the cases with PIV or PCI under the cutoff values that result in suboptimal surgery in PDS, we emphasize the importance of preoperative 


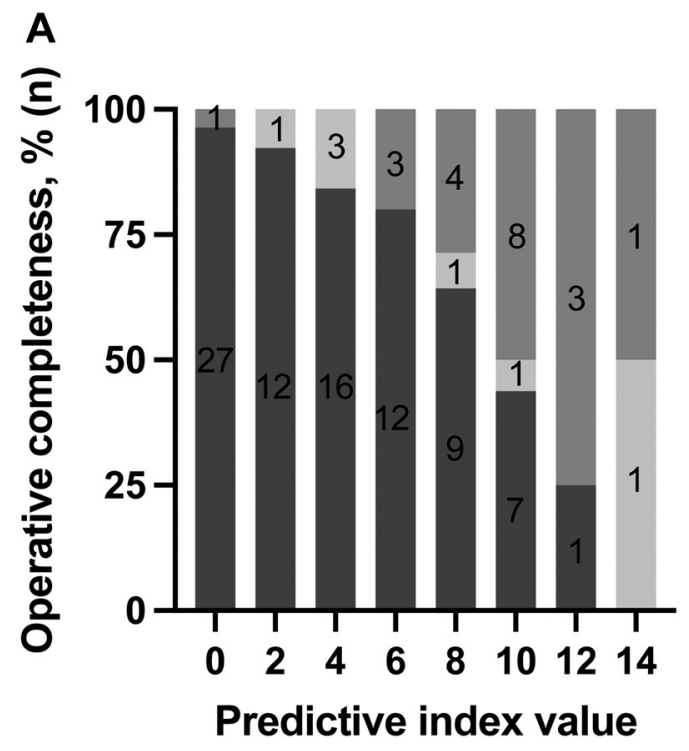

Complete

Optimal

Suboptimal

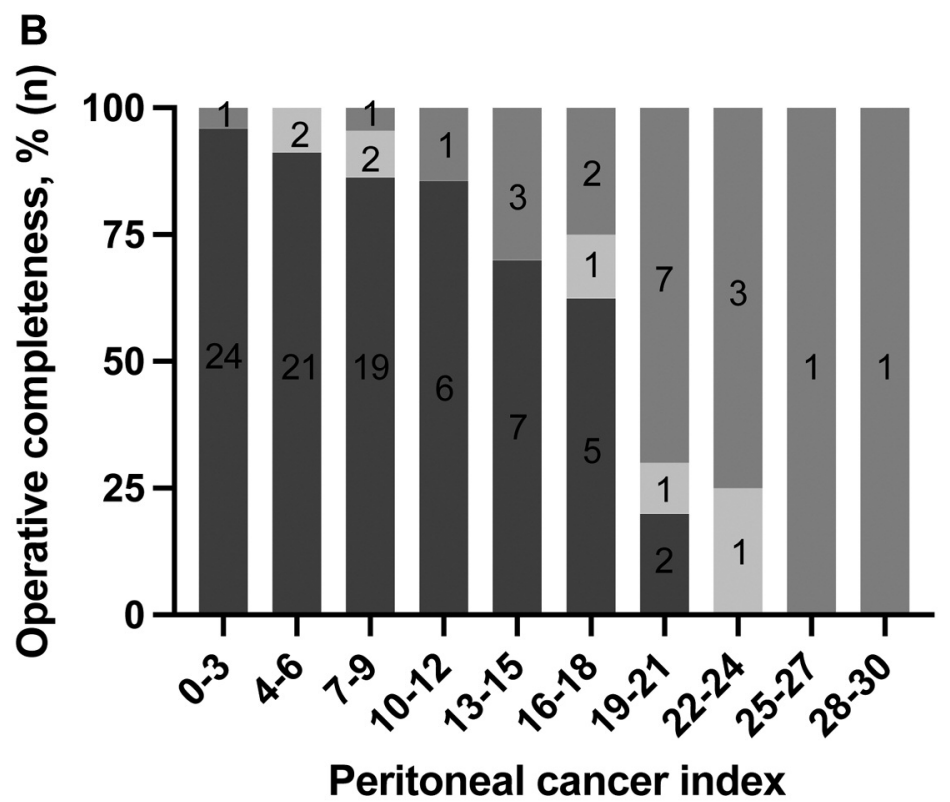

Figure 4. Operative outcomes and distribution of PIV and PCI. (A) The rate of complete surgery among patients with PIV $\leq 6$ is significantly higher than that among patients with PIV $\geq 8$ (89.3\% vs. $47.2 \%$ : $p<0.05)$. (B) Additionally, the rate of complete surgery among patients with PCI $\leq 12$ is also significantly higher than that among patients with $P C I \geq 13$ (90.9\% vs. $41.2 \%: p<0.05)$. No case had PCI $\geq 31$ points. Operative completeness is shown as the rate of residual tumor burden after primary debulking surgery (complete, optimal, or suboptimal surgery) presented as percentage (\%). The number of patients is presented in the column of each operative category (n). PIV: Predictive index value; PCI: peritoneal cancer index.

imaging examinations and developing a modified assessment system focusing on the small bowels and mesentery, such as the modified PIV (20). Further investigation is needed to establish a more precise preoperative scoring model that overcomes current limitations and improves the predictive ability by combining data from diagnostic operative features and preoperative imaging, which also might be helpful in the reduction of severe operative complications after PDS (29).

Recently, a new predictive model for complete PDS using artificial intelligence (AI) was reported by Laios et al. and yielded slightly higher accuracy of predicting complete PDS (30). Moreover, to integrate the reliability of these scoring systems at each institution, a free application for calculating PIV and PCI can be made available on the Internet (31). When AI can precisely assess PIV and PCI depending on a movie of laparoscopy for AOC and be linked to such freeaccess internet applications, these scoring systems become more generalized, and worldwide discussion of these scores for consensus could be held.

This study has some strengths. First, our study was one of the largest studies conducted with more than 100 eligible patients. Second, since we assessed both PIV and PCI, we can provide valuable data on the correlation and limitations of these scores. Contrarily, our research also has several limitations. First, because we gathered the data about disseminations by referring to operative and pathological records, we should recognize the possibility of an underestimated extent of disseminations. Second, because of the retrospective design, a selection bias can exist in the selection of therapeutic options, meaning that eligible patients tended to have a smaller degree of abdominal dissemination and those with larger disseminations on preoperative imaging examinations or diagnostic surgeries who were candidate for NAC+IDS could be substantively excluded. Third, since the skills and maximal effort in the surgical procedure are thought to be important for a safe and sufficient complete PDS (32), we should recognize the existence of this uncontrollable bias between each facility. In the consideration of over $70 \%$ of the complete PDS rate in our institutions, our results might be applied to other highvolume hospitals specializing in PDS. Finally, although PIV was originally designed for the laparoscopic prediction 
Table II. Clinicopathological features of patients under and above the cutoff value of PIV=6.

\begin{tabular}{|c|c|c|c|}
\hline Characteristics & PIV $\leq 6$ & PIV $\geq 8$ & $p$-Value \\
\hline Total cases, $\mathrm{n}$ & 75 & 36 & \\
\hline Age (years), mean \pm SD & $56.7 \pm 10.9$ & $57.0 \pm 10.3$ & 0.878 \\
\hline Preoperative CA125 level (IU/ml), mean \pm SD & $1,385.2 \pm 2,058.5$ & $2,956.1 \pm 4,315.6$ & $0.011 *$ \\
\hline Ascites on $\mathrm{CT}, \mathrm{n}(\%)$ & & & $0.017 *$ \\
\hline Negative & $26(34.7)$ & $4(11.1)$ & \\
\hline Positive & $49(65.3)$ & $32(88.9)$ & \\
\hline FIGO stage, n $(\%)$ & & & $<0.001^{*}$ \\
\hline IIIA & $17(22.7)$ & $0(0)$ & \\
\hline IIIB & $25(33.3)$ & $3(8.3)$ & \\
\hline IIIC & $33(44.0)$ & $32(88.9)$ & \\
\hline IVA & $0(0)$ & $1(2.8)$ & \\
\hline Histology, n (\%) & & & 0.155 \\
\hline High-grade serous carcinoma & $46(61.3)$ & $27(75.0)$ & \\
\hline Non-high-grade serous carcinoma & $29(38.7)$ & $9(25.0)$ & \\
\hline
\end{tabular}

${ }^{*} p<0.05$, Student's $t$-test or $\chi^{2}$ test. Continuous data were presented as means \pm standard deviation (SD); categorical data are presented as percent. PIV: Predictive index value; CT: computed tomography; FIGO: International Federation of Gynecology and Obstetrics staging system.

Table III. Clinicopathological features of patients under and above the cutoff value of $P C I=12$.

\begin{tabular}{|c|c|c|c|}
\hline Characteristics & $\mathrm{PCI} \leq 12$ & $\mathrm{PCI} \geq 13$ & $p$-Value \\
\hline Total cases, $\mathrm{n}$ & 77 & 34 & \\
\hline Age (years), mean \pm SD & $56.2 \pm 10.3$ & $58.1 \pm 11.4$ & 0.389 \\
\hline Preoperative CA125 level (IU/ml), mean \pm SD & $1,442.5 \pm 2,019.0$ & $2,918.8 \pm 4,486.4$ & $0.018^{*}$ \\
\hline Ascites in CT scan, $\mathrm{n}(\%)$ & & & $0.008^{*}$ \\
\hline Negative & $27(35.1)$ & $3(8.8)$ & \\
\hline Positive & $50(64.9)$ & $31(91.2)$ & \\
\hline FIGO stage, n (\%) & & & $<0.001 *$ \\
\hline IIIA & $17(22.1)$ & $0(0)$ & \\
\hline IIIB & $25(32.5)$ & $3(8.8)$ & \\
\hline IIIC & $35(45.4)$ & $30(88.2)$ & \\
\hline IVA & $0(0)$ & $1(3.0)$ & \\
\hline Histology, $\mathrm{n}(\%)$ & & & 0.204 \\
\hline High-grade serous carcinoma & $47(61.0)$ & $25(73.5)$ & \\
\hline Non-high-grade serous carcinoma & $30(39.0)$ & $9(26.5)$ & \\
\hline
\end{tabular}

${ }^{*} p<0.05$, Student's $t$-test or $\chi^{2}$ test. Continuous data are presented as means \pm standard deviation (SD); categorical data are presented as number (percentage). PCI: Peritoneal cancer index; CT: computed tomography; FIGO: International Federation of Gynecology and Obstetrics staging system.

Table IV. Suboptimal surgery cases with $P I V \leq 6$ or $P C I \leq 12$.

\begin{tabular}{lcccc}
\hline Case No. & Clinical stage & PIV & PCI & Reasons for suboptimal surgery \\
\hline 1 & IIIA & 0 & 3 & Massive infiltration of the para-aortic lymph node metastasis to the vertebra and renal vein \\
2 & IIIC & 6 & 9 & Deep infiltration of the tumor to the bladder \\
4 & IIIC & 6 & 13 & Massive infiltration of the tumor to the right ureter and retroperitoneum \\
4 & IIIC & 6 & 19 & Widespread mesenteric retraction and dissemination in the small bowel
\end{tabular}

PIV: Predictive index value; PCI: peritoneal cancer index. 


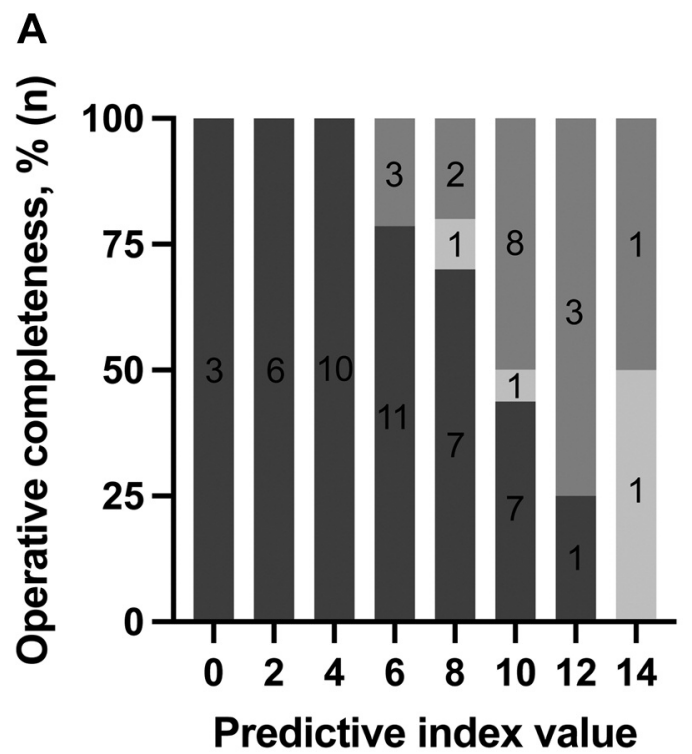

Complete

Optimal

Suboptimal
B

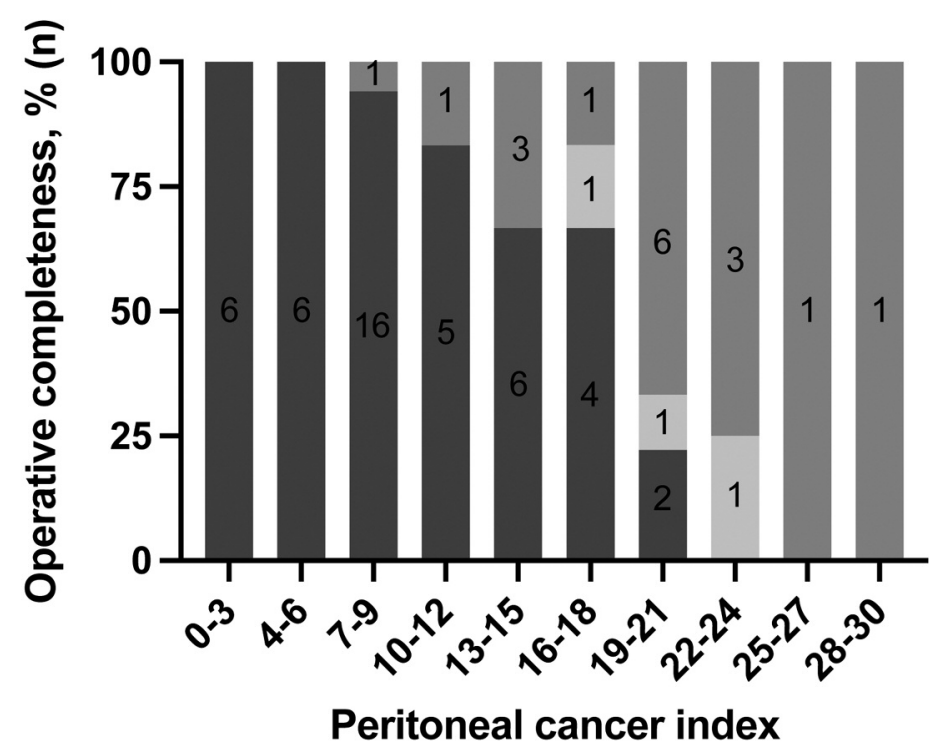

Figure 5. Operative outcomes and distribution of (A) PIV and (B) PCI in patients with FIGO stage IIIC. No case had PCI $\geq 31$ points. Operative completeness is shown as the rate of residual tumor burden after primary debulking surgery (complete, optimal, or suboptimal surgery) presented by percentage (\%) and the number of patients presented in the column of each operative category (n). PIV: Predictive index value; PCI: peritoneal cancer index; FIGO: International Federation of Gynecology and Obstetrics.

model of suboptimal surgery, we applied it in laparotomy. Therefore, a prospective study using PIV and PCI should be conducted to assess the relationship between the extent of disseminations in both laparoscopic and laparotomic findings and operative outcomes.

\section{Conclusion}

PIV and PCI, quantitative scoring systems for abdominal tumor disseminations, may be valuable in predicting operative completeness in AOC. Further multicenter prospective studies will be needed to validate the predictive values of these scores.

\section{Conflicts of Interest}

The Authors declare that they have no competing interests related to this study.

\section{Authors' Contributions}

SH initiated the concept, collected and analyzed the patient's data, and wrote the first draft. KU and SO initiated the concept, interpreted the data, supported the implementation of this study and revised the first draft. KO supported and gave comment on the statistical analysis and revised the draft. HK and TS supported the data collection and data analysis. MT, MS, KY, and HT also gave critical comments on the interpretation and revised the draft. YI and NY gave critical comments on the study design and interpretation and revised the draft. AO revised the draft and supervised this project. All Authors approved the final manuscript

\section{Acknowledgements}

The Authors would like to thank Enago (www.enago.jp) for the English language review.

\section{References}

1 Torre LA, Bray F, Siegel RL, Ferlay J, Lortet-Tieulent J and Jemal A: Global cancer statistics, 2012. CA Cancer J Clin 65(2): 87-108, 2015. PMID: 25651787. DOI: 10.3322/caac.21262

2 Machida H, Matsuo K, Yamagami W, Ebina Y, Kobayashi Y, Tabata T, Kanauchi M, Nagase S, Enomoto T and Mikami M: Trends and characteristics of epithelial ovarian cancer in Japan between 2002 and 2015: A JSGO-JSOG joint study. Gynecol Oncol 153(3): 589-596, 2019. PMID: 30905436. DOI: 10.1016/ j.ygyno.2019.03.243 
3 Armstrong DK, Alvarez RD, Bakkum-Gamez JN, Barroilhet L, Behbakht K, Berchuck A, Chen LM, Cristea M, DeRosa M, Eisenhauer EL, Gershenson DM, Gray HJ, Grisham R, Hakam A, Jain A, Karam A, Konecny GE, Leath CA, Liu J, Mahdi H, Martin L, Matei D, McHale M, McLean K, Miller DS, O’Malley DM, Percac-Lima S, Ratner E, Remmenga SW, Vargas R, Werner TL, Zsiros E, Burns JL and Engh AM: Ovarian cancer, version 2.2020, NCCN clinical practice guidelines in oncology. J Natl Compr Canc Netw 19(2): 191-226, 2021. PMID: 33545690. DOI: $10.6004 /$ jncen.2021.0007

4 Vergote I, Tropé CG, Amant F, Kristensen GB, Ehlen T, Johnson $\mathrm{N}$, Verheijen RH, van der Burg ME, Lacave AJ, Panici PB, Kenter GG, Casado A, Mendiola C, Coens C, Verleye L, Stuart GC, Pecorelli S, Reed NS, European Organization for Research and Treatment of Cancer-Gynaecological Cancer Group and NCIC Clinical Trials Group: Neoadjuvant chemotherapy or primary surgery in stage IIIC or IV ovarian cancer. $\mathrm{N}$ Engl $\mathrm{J}$ Med 363(10): 943-953, 2010. PMID: 20818904. DOI: 10.1056/NEJMoa0908806

5 Kehoe S, Hook J, Nankivell M, Jayson GC, Kitchener H, Lopes T, Luesley D, Perren T, Bannoo S, Mascarenhas M, Dobbs S, Essapen S, Twigg J, Herod J, McCluggage G, Parmar M and Swart AM: Primary chemotherapy versus primary surgery for newly diagnosed advanced ovarian cancer (CHORUS): an open-label, randomised controlled, non-inferiority trial. Lancet 386(9990): 249-257, 2015. PMID: 26002111. DOI: 10.1016/S0140-6736(14)62223-6

6 Onda T, Satoh T, Ogawa G, Saito T, Kasamatsu T, Nakanishi T, Mizutani T, Takehara K, Okamoto A, Ushijima K, Kobayashi H, Kawana K, Yokota H, Takano M, Kanao H, Watanabe Y, Yamamoto K, Yaegashi N, Kamura T, Yoshikawa H and Japan Clinical Oncology Group: Comparison of survival between primary debulking surgery and neoadjuvant chemotherapy for stage III/IV ovarian, tubal and peritoneal cancers in phase III randomised trial. Eur J Cancer 130: 114-125, 2020. PMID: 32179446. DOI: 10.1016/j.ejca.2020.02.020

7 Fagotti A, Ferrandina MG, Vizzielli G, Pasciuto T, Fanfani F, Gallotta V, Margariti PA, Chiantera V, Costantini B, Gueli Alletti S, Cosentino F and Scambia G: Randomized trial of primary debulking surgery versus neoadjuvant chemotherapy for advanced epithelial ovarian cancer (SCORPION-NCT01461850). Int J Gynecol Cancer 30(11): 1657-1664, 2020. PMID: 33028623. DOI: 10.1136/ijgc-2020-001640

8 Reuss A, du Bois A, Harter P, Fotopoulou C, Sehouli J, Aletti G, Guyon F, Greggi S, Mosgaard BJ, Reinthaller A, Hilpert F, Schade-Brittinger C, Chi DS and Mahner S: TRUST: Trial of Radical Upfront Surgical Therapy in advanced ovarian cancer (ENGOT ov33/AGO-OVAR OP7). Int J Gynecol Cancer 29(8): 1327-1331, 2019. PMID: 31420412. DOI: 10.1136/ijgc-2019000682

9 Jiang R, Zhu J, Kim JW, Liu J, Kato K, Kim HS, Zhang Y, Zhang P, Zhu T, Aoki D, Yu A, Chen X, Wang X, Zhu D, Zhang W, Jia H, Shi T, Gao W, Yin S, Feng Y, Xiang L, Okamoto A and Zang R: Study of upfront surgery versus neoadjuvant chemotherapy followed by interval debulking surgery for patients with stage IIIC and IV ovarian cancer, SGOG SUNNY (SOC-2) trial concept. J Gynecol Oncol 31(5): e86, 2020. PMID: 32808504. DOI: 10.3802/jgo.2020.31.e86

10 Odajima S, Ueda K, Hosoya S, Tomita K, Kato S, Shoburu Y, Kawabata A, Iida Y, Yanaihara N and Okamoto A: Clinical availability of tumour biopsy using diagnostic laparoscopy for advanced ovarian cancer. In Vivo 35(6): 3325-3331, 2021. PMID: 34697165. DOI: 10.21873/invivo.12629

11 Fagotti A, Ferrandina G, Fanfani F, Ercoli A, Lorusso D, Rossi $\mathrm{M}$ and Scambia G: A laparoscopy-based score to predict surgical outcome in patients with advanced ovarian carcinoma: a pilot study. Ann Surg Oncol 13(8): 1156-1161, 2006. PMID: 16791447. DOI: 10.1245/ASO.2006.08.021

12 Tentes AA, Tripsiannis G, Markakidis SK, Karanikiotis CN, Tzegas G, Georgiadis G and Avgidou K: Peritoneal cancer index: a prognostic indicator of survival in advanced ovarian cancer. Eur J Surg Oncol 29(1): 69-73, 2003. PMID: 12559080. DOI: 10.1053 /ejso.2002.1380

13 van de Vrie R, Rutten MJ, Asseler JD, Leeflang MM, Kenter GG, Mol BWJ and Buist M: Laparoscopy for diagnosing resectability of disease in women with advanced ovarian cancer. Cochrane Database Syst Rev 3: CD009786, 2019. PMID: 30907434. DOI: 10.1002/14651858.CD009786.pub3

14 Saito M, Odajima S, Ezawa M, Iida Y, Ueda K, Yanaihara N, Tanabe H, Takano H, Yamada K and Okamoto A: Neoadjuvant chemotherapy for patients with diaphragmatic lesions: a prognostic postoperative analysis. In Vivo 34(3): 1277-1281, 2020. PMID: 32354919. DOI: 10.21873/invivo.11902

15 Sugarbaker PH and Jablonski KA: Prognostic features of 51 colorectal and 130 appendiceal cancer patients with peritoneal carcinomatosis treated by cytoreductive surgery and intraperitoneal chemotherapy. Ann Surg 221(2): 124-132, 1995. PMID: 7857141. DOI: 10.1097/00000658-199502000-00002

16 Chéreau E, Ballester M, Selle F, Cortez A, Daraï E and Rouzier $\mathrm{R}$ : Comparison of peritoneal carcinomatosis scoring methods in predicting resectability and prognosis in advanced ovarian cancer. Am J Obstet Gynecol 202(2): 178.e1-178.e10, 2010. PMID: 20113693. DOI: 10.1016/j.ajog.2009.10.856

17 Gilly FN, Cotte E, Brigand C, Monneuse O, Beaujard AC, Freyer $\mathrm{G}$ and Glehen $\mathrm{O}$ : Quantitative prognostic indices in peritoneal carcinomatosis. Eur J Surg Oncol 32(6): 597-601, 2006. PMID: 16617003. DOI: 10.1016/j.ejso.2006.03.002

18 Chesnais M, Lecuru F, Mimouni M, Ngo C, Fauconnier A and Huchon C: A pre-operative predictive score to evaluate the feasibility of complete cytoreductive surgery in patients with epithelial ovarian cancer. PLoS One 12(11): e0187245, 2017. PMID: 29117194. DOI: 10.1371/journal.pone. 0187245

19 Llueca A, Climent MT, Escrig J, Carrasco P, Serra A and MUAPOS working group (Multidisciplinary Unit of Abdominal Pelvic Oncology Surgery): Validation of three predictive models for suboptimal cytoreductive surgery in advanced ovarian cancer. Sci Rep 11(1): 8111, 2021. PMID: 33854085. DOI: 10.1038/s41598021-86928-2

20 Brun JL, Rouzier R, Uzan S and Daraï E: External validation of a laparoscopic-based score to evaluate resectability of advanced ovarian cancers: clues for a simplified score. Gynecol Oncol 110(3): 354-359, 2008. PMID: 18572226. DOI: 10.1016/j.ygyno.2008. 04.042

21 Muallem MZ, Sehouli J, Richter R, Babayeva A, Gasimli K and Parashkevova A: Pre-operative serum CA125, peritoneal cancer index and intra-operative mapping score as predictors of surgical results in primary epithelial ovarian cancer. Int J Gynecol Cancer 30(1): 62-66, 2020. PMID: 31744887. DOI: 10.1136/ijgc-2019000778

22 Fagotti A, Ferrandina G, Fanfani F, Garganese G, Vizzielli G, Carone V, Salerno MG and Scambia G: Prospective validation of 
a laparoscopic predictive model for optimal cytoreduction in advanced ovarian carcinoma. Am J Obstet Gynecol 199(6): 642.e1642.e6, 2008. PMID: 18801470. DOI: 10.1016/j.ajog.2008.06.052

23 Feng Z, Wen H, Jiang Z, Liu S, Ju X, Chen X, Xia L, Xu J, Bi $\mathrm{R}$ and $\mathrm{Wu} \mathrm{X}$ : A triage strategy in advanced ovarian cancer management based on multiple predictive models for R0 resection: a prospective cohort study. J Gynecol Oncol 29(5): e65, 2018. PMID: 30022629. DOI: 10.3802/jgo.2018.29.e65

24 Llueca A, Escrig $J$ and MUAPOS working group (Multidisciplinary Unit of Abdominal Pelvic Oncology Surgery): Prognostic value of peritoneal cancer index in primary advanced ovarian cancer. Eur J Surg Oncol 44(1): 163-169, 2018. PMID: 29198495. DOI: $10.1016 /$ j.ejso.2017.11.003

25 Elzarkaa AA, Shaalan W, Elemam D, Mansour H, Melis M, Malik E and Soliman AA: Peritoneal cancer index as a predictor of survival in advanced stage serous epithelial ovarian cancer: a prospective study. J Gynecol Oncol 29(4): e47, 2018. PMID: 29770618. DOI: 10.3802/jgo.2018.29.e47

26 Gouy S, Belghiti J, Uzan C, Canlorbe G, Gauthier T and Morice P: Accuracy and reproducibility of the peritoneal cancer index in advanced ovarian cancer during laparoscopy and laparotomy. Int J Gynecol Cancer 23(9): 1699-1703, 2013. PMID: 24100589. DOI: 10.1097/IGC.0b013e3182a616a7

27 Zeff N: Role of laparoscopy in initial tumour staging in advanced epithelial ovarian cancer: A systematic review. Pleura Peritoneum 3(1): 20180106, 2018. PMID: 30911654. DOI: 10.1515/pp-2018-0106

28 Fagotti A, Perelli F, Pedone L and Scambia G: Current recommendations for minimally invasive surgical staging in ovarian cancer. Curr Treat Options Oncol 17(1): 3, 2016. PMID: 26739150. DOI: 10.1007/s11864-015-0379-8
29 Collins A, Spooner S, Horne J, Chainrai M, Runau F, Bourne T, Moss EL, Davies Q, Chattopadhyay S and Bharathan R: Perioperative variables associated with prolonged intensive care stay following cytoreductive surgery for ovarian cancer. Anticancer Res 41(6): 3059-3065, 2021. PMID: 34083298. DOI: 10.21873/anticanres.15089

30 Laios A, Gryparis A, DeJong D, Hutson R, Theophilou G and Leach C: Predicting complete cytoreduction for advanced ovarian cancer patients using nearest-neighbor models. J Ovarian Res 13(1): 117, 2020. PMID: 32993745. DOI: 10.1186/s13048020-00700-0

31 Villeneuve L, Thivolet A, Bakrin N, Mohamed F, Isaac S, Valette PJ, Glehen O, Rousset P and BIG-RENAPE and RENAPE Working Groups: A new internet tool to report peritoneal malignancy extent. PeRitOneal MalIgnancy Stage Evaluation (PROMISE) application. Eur J Surg Oncol 42(6): 877-882, 2016. PMID: 27067193. DOI: 10.1016/j.ejso.2016.03.015

32 Egger EK, Kohls N, Stope MB, Condic M, Keyver-Paik MD, KÖnsgen D, Hilbert T, Klaschik S, Exner D, Vilz T and Mustea A: Risk factors for severe complications in ovarian cancer surgery. In Vivo 34(6): 3361-3365, 2020. PMID: 33144443. DOI: 10.21873 /invivo.12174
Received October 24, 2021

Revised November 11, 2021 Accepted November 12, 2021 